\title{
857 SELECTIVE TREG DEPLETION IN SOLID TUMORS WITH ALD2510, A NOVEL HUMANIZED CD25-SPECIFIC, IL-2 SPARING MONOCLONAL ANTIBODY
}

${ }^{1}$ Jemila Houacine, ${ }^{2}$ Anne Marie-Cardine, ${ }^{1}$ Aude Le Roy, ${ }^{3}$ Jérôme Giustiniani, ${ }^{1}$ Riad Abes, ${ }^{4}$ Anne-Sophie Chrétien, ${ }^{4}$ Stéphane Fattori, ${ }^{4}$ Laurent Gorvel, ${ }^{2}$ Armand Bensussan, ${ }^{4}$ Daniel Olive, ${ }^{1}$ Arnaud Foussat*. 'A Alderaan Biotechnology, Paris, France; ${ }^{2}$ INSERM U976, Paris, France; ${ }^{3}$ Inserm U955, Créteil, France; ${ }^{4}$ Inserm UMR1068, Institut Paoli Calmettes, Marseille, France

Background Regulatory $\mathrm{T}$ cells (Tregs) inhibit immune responses in solid cancers using cell-cell contacts and antiinflammatory cytokine release. Also, due to high and constitutive levels of IL2Ralpha chain (CD25) expression, Tumor infiltrating (TIL)-Tregs cells preferably consume local Interleukin-2 (IL2), thus depriving conventional $\mathrm{T}$ cells from IL2-induced activation and proliferation. Therefore, the selective depletion of TIL-Tregs using therapeutic antibodies targeting CD25 represents a promising strategy to unleash tumor-specific immune responses in solid cancers.

Methods CD25 expression was evaluated by flow and mass cytometry on $\mathrm{T}$-cell subsets from tumor biopsies collected in patients with various solid cancers (Breast, Endometrial and Cervix). ALD2510 potency was demonstrated in vitro and in vivo in human CD25 Knock-In huGEMM (huCD25-KI) MC38-bearing mice and in CD34+ humanized NSG mice grafted with human cancer cell lines (MDA-MB-231 and HT29).

Results In tumor biopsies, CD25 is highly and homogeneously expressed by TIL-Tregs, while being much less expressed by only a fraction of conventional CD4 $+\mathrm{T}$ cells and barely expressed by TIL-CD8 + cells. This confirms CD25 as the most selective marker to target TIL-Tregs in cancer patients.In vitro, ALD2510 shows potent ADCC and ADCP as well as strong Treg depletion capacity. Importantly, CD8+ and CD4+ conventional $\mathrm{T}$ cells are not impacted by ALD2510 even after activation confirming ALD2510 ability to selectively deplete Tregs. Accordingly, ALD2510 neither blocks IL-2 binding to CD25 nor inhibits IL-2 induced proliferation of activated T cells. In CD34+-humanized mice, ALD2510 efficiently depletes human Tregs but spares conventional T cells. Also, in the MC38 model in huCD25-KI mice, ALD2510 shows a strong anti-tumor activity as a single agent with $60 \%$ overall tumor growth inhibition together with massive Treg depletion 7 days after a single administration. In addition, combination of ALD2510 with anti-PD1 leads to complete tumor regression and strong activation of conventional $\mathrm{T}$ cells. Importantly, Basiliximab, a CD25-specific IL-2 blocking antibody, although efficient at depleting Treg cells, did not impact tumor growth, thus demonstrating that the IL-2 sparing feature of ALD2510 is critical to elicit anti-tumour response in vivo.

Conclusions This preclinical data package supports CD25 as a potent and selective Treg marker allowing Tregs depletion while sparing conventional T cells. In this context, ALD2510, a novel humanized CD25-specific and IL-2 sparing antibody presents all the required attributes for selective and efficient TIL-Tregs depletion, making it a promising drug candidate to treat a broad range of solid tumor patients.

Ethics Approval The studies involving human material were approved by the ethical committee "Comité de Protection des Personnes Sud Méditerranée » under approval numbers 1362 and 1048. All participants gave informed consent before taking part. 